\title{
Comparison of Antimicrobial Resistance of Acinetobacter baumannii Clinical Isolates from Shanghai and Hong Kong
}

\author{
Thomas K.W. Ling ${ }^{a}$ Chun Mei Ying $^{b}$ Ching Ching Lee ${ }^{a}$ Zhi Kai Liu ${ }^{a}$ \\ a Department of Microbiology, The Chinese University of Hong Kong, Prince of Wales Hospital, \\ Hong Kong, SAR, and ${ }^{b}$ Department of Microbiology, Shanghai Second Medical University, Renji Hospital, \\ Shanghai, China
}

\section{Key Words}

Antimicrobial resistance $\cdot$ Acinetobacter baumannii .

Shanghai $\cdot$ Hong Kong

\begin{abstract}
Objective: To compare the antimicrobial resistance patterns of Acinetobacter baumannii isolates from Shanghai and Hong Kong. Materials and Methods: A total of $212 A$. baumannii strains of one isolate per patient were collected from Shanghai and Hong Kong from August 2002 to August 2003 that were tested against $15 \mathrm{com}$ monly used antimicrobial agents by the agar dilution method according to the NCCLS guidelines. Results: Most $\beta$-lactams showed no significant increase in activity after adding $\beta$-lactamase inhibitors. The resistance rates of the isolates against ticarcillin-clavulanate, piperacillin-tazobactam and ampicillin-sulbactam were for Shanghai 74.9, 70.9, 69.1\% and Hong Kong 24.3, 18.9, $13.5 \%$, respectively. Only cefoperazone-sulbactam showed a significant increase in activity against both Shanghai and Hong Kong strains, as the resistance rates dropped from 93.7 to $8.6 \%$ and 83.8 to $5.4 \%$, respectively. The resistance rates of ceftazidime, cefepime, and gentamicin against Shanghai strains were 69.7, 72.0, $73.7 \%$ and Hong Kong strains 69.7, 29.7, 18.9\%, respectively. About $65 \%$ of Shanghai strains were found to be
\end{abstract}

amikacin-resistant, however, all Hong Kong strains were sensitive. Fluoroquinolones including ciprofloxacin and levofloxacin had resistance rates over $60 \%$ against Shanghai strains, but only $13.5 \%$ against Hong Kong strains. Shanghai strains had imipenem and meropenem resistance rate of $6.3 \%$. Though $10.8 \%$ Hong Kong strains were resistant to meropenem, only $2.7 \%$ of them were resistant to imipenem. Conclusion: A. baumannii isolated from Shanghai were more resistant to all drugs except meropenem than Hong Kong isolates. The results indicate a need for measures to control the abuse of antibiotic usage in order to prevent the emergence of more multidrug-resistant isolates in both cities.

Copyright $\odot 2005$ S. Karger AG, Basel

\section{Introduction}

Acinetobacter spp. are a group of important opportunistic pathogens. They are oxidase-negative, non-fermentative gram-negative rods that can cause a wide variety of nosocomial infections among immunocompromised patients or patients with severe underlying diseases especially in the intensive care units [1-4]. Acinetobacter baumannii is the most frequently isolated species in the hospital environment. During the early 1980s, fluoroquinolones were active against $A$. baumannii infections.

\section{KARGER}

Fax +4161306 1234 E-Mail karger@karger.ch www.karger.com
(C) 2005 S. Karger AG, Basel

$1011-7571 / 05 / 0145-0338 \$ 22.00 / 0$

Accessible online at:

www.karger.com/mpp
Dr. Thomas K.W. Ling

Department of Microbiology, The Chinese University of Hong Kong Prince of Wales Hospital

Hong Kong, SAR (China)

Tel. +852 26322307, Fax +852 26451256, E-Mail lingt@cuhk.edu.hk 
Table 1. MIC and the percentages of resistance $(\% \mathrm{R})$ of $A$. baumannii isolated from Shanghai and Hong Kong

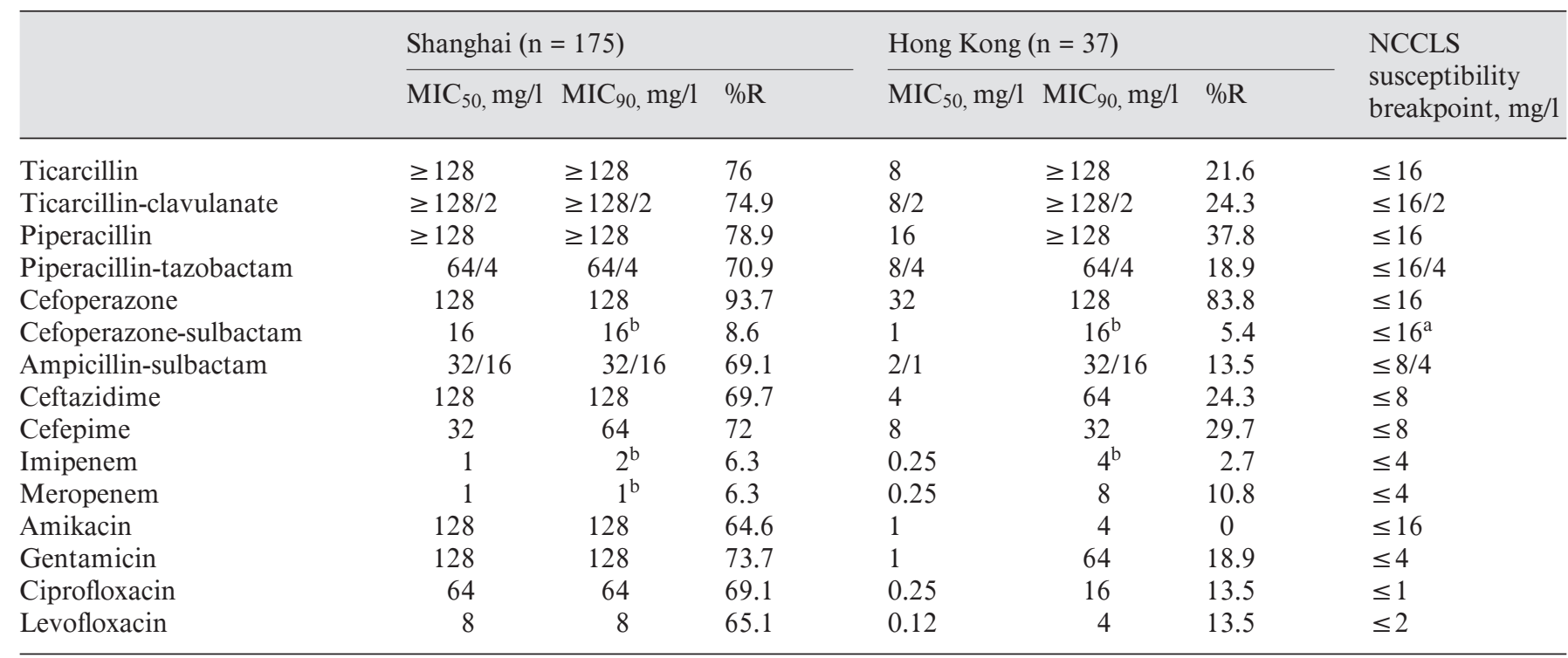

${ }^{a}$ The susceptibility breakpoint of cefoperazone-sulbactam $\leq 16 \mathrm{mg} / \mathrm{l}$ (not listed in the NCCLS).

$\mathrm{b}$ The $\mathrm{MIC}_{90}$ were equivalent to or less than their NCCLS susceptibility breakpoints. There should be $90 \%$ or more $A$. baumannii isolates susceptible to this antimicrobial agent.

However, more and more $A$. baumannii clinical isolates have become resistant. Carbapenems such as imipenem and meropenem are the most effective antimicrobial agents against $A$. baumannii $[5,6]$. There are only a few reports about the antimicrobial susceptibility results of $A$. baumannii from China including two major large cities, Shanghai and Hong Kong [7]. Our study aimed to compare the antimicrobial resistance patterns of A. baumannii isolates from Shanghai and Hong Kong, that could be important for local surveillance and determination of the most effective therapy.

\section{Materials and Methods}

A total of 212 clinical isolates of $A$. baumannii (one isolate per patient) were collected from Renji Hospital, Shanghai $(n=175)$ and the Prince of Wales Hospital, Hong Kong $(n=37)$ between August 2002 and August 2003. The Shanghai isolates were collected from various clinical specimens including sputum, wound, abscess, bile, urine, and blood cultures. The Hong Kong isolates were collected from blood cultures only. All isolates were identified by the API identification system (API Systems, Vercieu, France) and other standard biochemical tests [8].

Fifteen antimicrobial agents including carbapenems, $\beta$-lactams, $\beta$-lactamase inhibitor combinations, aminoglycosides, cephems and fluoroquinolones were tested against $A$. baumannii isolates. Minimum inhibitory concentrations (MICs) were determined by the agar dilution method using Mueller-Hinton agar (Oxoid, UK) according to the National Committee for Clinical Laboratory Standards (NCCLS) [9]. A multipoint inoculator (Mast, UK) was used to deliver $10^{4}$ colony forming units (CFUs) per spot onto the agar plates with different antimicrobial agents. All plates were incubated at $37^{\circ} \mathrm{C}$ for $18 \mathrm{~h}$. MICs (mg/l) were defined as the concentrations at which no colony was detected on the agar. Escherichia coli ATCC 25922, ATCC 35218 and Pseudomonas aeruginosa ATCC 27853 were used as controls. MICs defined as NCCLS intermediate category were grouped as resistant in this study.

\section{Results}

$\mathrm{MIC}_{50}, \mathrm{MIC}_{90}$ and the percentages of resistance of the A. baumannii isolates from Shanghai and Hong Kong are listed in table 1. $\beta$-Lactamase antibiotics except cefoperazone showed no significant increase in activity after adding inhibitors such as clavulanate and tazobactam. The resistance rates to ticarcillin-clavulanate, piperacillintazobactam and ampicillin-sulbactam against Shanghai isolates were $74.9,70.9,69.1 \%$ and Hong Kong isolates were $24.3,18.9,13.5 \%$, respectively, with $\mathrm{MIC}_{90}$ $\geq 64 \mathrm{mg} / \mathrm{l}$. Cefoperazone showed a significant increase in activity after adding sulbactam. The resistance rates of the Shanghai isolates and the Hong Kong isolates dropped from 93.7 to $8.6 \%$ and from 83.8 to $5.4 \%$, respec- 
tively. $\mathrm{MIC}_{50}$ and $\mathrm{MIC}_{90}$ also decreased from 128 to 16 $\mathrm{mg} / \mathrm{l}$. The resistance rates to ceftazidime and cefepime against the Shanghai and Hong Kong strains were about 70 and $25 \%$, respectively $\left(\mathrm{MIC}_{90}=128\right.$ and $64 \mathrm{mg} / \mathrm{l}$, and $\mathrm{MIC}_{90}=64$ and $\left.32 \mathrm{mg} / \mathrm{l}\right)$.

Among all the antimicrobial agents tested, carbapenems including imipenem and meropenem showed high activity against the Shanghai isolates with $\mathrm{MIC}_{90}$ $\leq 2 \mathrm{mg} / \mathrm{l}$, of which only $6.3 \%$ were resistant. Approximately, $11 \%$ of Hong Kong strains were resistant to meropenem $\left(\mathrm{MIC}_{90}=8 \mathrm{mg} / \mathrm{l}\right)$, but only $2.7 \%$ were resistant to imipenem $\left(\mathrm{MIC}_{90}=4 \mathrm{mg} / \mathrm{l}\right)$. Approximately $65 \%$ Shanghai strains were amikacin-resistant $\left(\mathrm{MIC}_{90}=\right.$ $128 \mathrm{mg} / \mathrm{l}$ ), but all Hong Kong isolates were sensitive. The Shanghai strains were threefold more resistant against gentamicin than the Hong Kong strains with rates of 73.7 and $18.9 \%$, respectively. Fluoroquinolones including ciprofloxacin and levofloxacin were fourfold less active in Shanghai than Hong Kong isolates $(60 \%$ resistance for Shanghai and only $13.5 \%$ for Hong Kong isolates).

\section{Discussion}

Acinetobacter spp. are widely distributed in both natural and hospital environments [10]. This group of organisms are the second most commonly isolated nonfermenters following $P$. aeruginosa. They are able to survive on moist and dry surfaces, and may also be present on healthy human skin [11]. The organisms are associated with hospital-acquired infections and most frequently observed during the warm season. The involved organs/tissues include the respiratory and urinary tracts and wounds (including catheter sites). The infection may progress to septicemia [11-13]. Risk factors for Acinetobacter spp. include inappropriate antibiotic treatment, and/or surgery, instrumentation, and stay in intensive care units. Acinetobacter spp. isolates are more often colonizers than infecting agents [14]. In recent years, multidrug-resistant Acinetobacter spp. isolates are frequently found all around the world [15].

Carbapenems including imipenem and meropenem were very active against the $A$. baumannii isolates from both cities with more than $90 \%$ of the isolates susceptible. Only 6.3\% Shanghai strains and 2.7\% Hong Kong strains were resistant to imipenem. However, about 10\% Hong Kong strains were resistant to meropenem. It was the only antimicrobial agent to which Hong Kong isolates had a higher antimicrobial resistance rate compared to Shang- hai strains. This may be due to three Hong Kong strains in the NCCLS intermediate category that were classified as resistant in the study.

Most $\beta$-lactamase inhibitor combinations except cefoperazone-sulbactam did not significantly improve the antimicrobial activity including ticarcillin-clavulanate, piperacillin-tazobactam and ampicillin-sulbactam [16]. All the resistance rates were over 70\% against the Shanghai isolates and ranged between 15 and 20\% against the Hong Kong isolates. Sulbactam greatly improved the activity of cefoperazone, as the resistance rates of cefoperazone-sulbactam decreased to 8.6 and $5.4 \%$ against the Shanghai and Hong isolates, respectively. Sulbactam has been shown to be a superior $\beta$-lactamase inhibitor compared with clavulanic acid and tazobactam against the multiresistant $A$. baumannii isolates [17, 18].

To deal with the increasing antimicrobial resistance of A. baumannii, measures for controlling overuse and abuse of antibiotics should be instituted in these hospitals. Antibiotics should only be prescribed if an infection is highly suspected. Physicians should choose an older generation of antibiotic according to the antimicrobial susceptibility results and reserve the powerful newer antibiotics. If the culture results are shown to be negative and no sign of infection, antibiotic therapy should be discontinued.

In a future study, the molecular epidemiology of the resistant strains from both cities such as three meropenem-resistant strains from Hong Kong should be investigated. Amplified ribosomal DNA restriction analysis and pulse-field gel electrophoresis can be used.

\section{Conclusion}

Imipenem, meropenem and cefoperazone-sulbactam were the most effective antimicrobial agents against the A. baumannii isolates from Shanghai and Hong Kong. Amikacin showed exceptional high activity against the isolates from Hong Kong. The Shanghai strains were more resistant to all drugs except meropenem than their Hong Kong counterparts.

\section{Acknowledgments}

We are grateful to the technical staff of the Department of Microbiology, the Prince of Wales Hospital, Hong Kong for their kind assistance. 


\section{References}

$\checkmark 1$ Theaker C, Azadian B, Soni N: The impact of Acinetobacter baumannii in the intensive care unit. Anaesthesia 2003;58:271-274.

-2 El Shafie SS, Alishaq M, Leni Garcia M: Investigation of an outbreak of multidrug-resistant Acinetobacter baumannii in trauma intensive care unit. J Hosp Infect 2004;56:101-105.

$\checkmark 3$ Herruzo R, de la Cruz J, Fernandez-Acenero MJ, Garcia-Caballero J: Two consecutive outbreaks of Acinetobacter baumannii 1-a in a burn Intensive Care Unit for adults. Burns 2004;30:419-423.

4 Rello J: Acinetobacter baumannii infections in the ICU: customerization is the key. Chest 1999; 115:1226-1229.

5 Jones ME, Thornsberry C, Livermore DM, Sahm DF: Prevalence of Acinetobacter spp. isolates with reduced sensitivity to imipenem, as determined by a USA-wide electronic surveillance network. J Antimicrob Chemother 1999;43:429-431.

$\checkmark 6$ Afzal MS, Livermore DM: Worldwide emergence of carbapenem-resistant Acinetobacter spp. J Antimicrob Chemother 1998;41:576577.

7 Ling JM, Ng TK, Cheng AF, Norrby SR: Susceptibilities to 23 antimicrobial agents and beta-lactamase production of blood culture isolates of Acinetobacter sp. in Hong Kong. Scand J Infect Dis 1996;101:21-25.
8 Murray PR, Barron EJ, Pfaller MA, Tenover FC, Yolken RH (eds): Manual of Clinical Microbiology, ed 7. Washington, American Society for Microbiology, 1999.

9 National Committee for Clinical Laboratory Standards: Methods for Dilution Antimicrobial Susceptibility Tests for Bacteria That Grow Aerobically, ed 4. Approved standard M7-A4. Wayne, National Committee for Clinical Laboratory Standards, 1997.

10 Bergogne-Berezin E, Towner KJ:Acinetobacter spp. as nosocomial pathogens: microbiological, clinical, and epidemiological features. Clin Microbiol Rev 1996;9:148-165.

11 Berlau J, Aucken HM, Malnick H, Pitt TL: Distribution of Acinetobacter spp. on skin of healthy humans. Eur J Clin Microbiol Infect Dis 1999; 18:179-183.

12 Cisneros JM, Reyes MJ, Pachon J, Becerril B, Caballero FJ, Garcia-Garmendia JL, Ortiz C, Cobacho R: Bacteremia due to Acinetobacter baumannii: epidemiology, clinical findings, and prognostic features. Clin Infect Dis 1996; 22:1026-1032.

13 Husni RN, Goldstein LS, Arroliga AC, Hall GS, Fatica C, Stoller JKG, Gordon SM: Risk factors for an outbreak of multi-drug-resistant Acinetobacter nosocomial pneumonia among intubated patients. Chest 1999;115:13781382.
14 Wisplinghoff $\mathrm{H}$, Perbix W, Seifert H: Risk factors for nosocomial bloodstream infections due to Acinetobacter baumannii: a case-control study of adult burn patients. Clin Infect Dis 1999;28:59-66.

15 Timsit JF, Garrait V, Misset B, Goldstein FW, Renaud B, Carlet $\mathrm{J}$ : The digestive tract is a major site for Acinetobacter baumannii colonization in intensive care unit patients. J Infect Dis 1993; 168:1336-1337.

16 Dy ME, Nord JA, Labombardi VJ, Kislak JW: The emergence of resistant strains of Acinetobacter baumannii: clinical and infection control implications. Infect Control Hosp Epidemiol 1999;20:565-567.

17 Higgins PG, Wisplinghoff H, Stefanik D, Seifert $\mathrm{H}$ : In vitro activities of the beta-lactamase inhibitors clavulanic acid, sulbactam, and tazobactam alone or in combination with betalactams against epidemiologically characterized multi-drug resistant Acinetobacter baumannii strains. Antimicrob Agents Chemother 2004; 48:1586-1592.

18 Wang FD, Lin ML, Lee WS, Liu CY: In vitro activities of beta-lactam antibiotics alone and in combination with sulbactam against Gramnegative bacteria. Int $\mathrm{J}$ Antimicrob Agents 2004;23:590-595. 